\title{
EAS longitudinal development distribution parameters for different extrapo- lations of the nuclei intaraction cross section to the very high energy domain
}

\author{
Zbigniew Plebaniak ${ }^{1, \star}$ and Tadeusz Wibig ${ }^{1,2, \star \star}$ \\ ${ }^{1}$ National Centre for Nuclear Research, Astrophysics Division, Cosmic Ray Laboratory, \\ ul. 28 Pułku Strzelców Kaniowskich 69, 90-558 Łód'z, Poland \\ ${ }^{2}$ Faculty of Physics and Applied Informatics, University of $Ł o ́ d ' z$, \\ ul. Pomorska 149/153, 90-236 Łód'z, Poland
}

\begin{abstract}
Determination of the primary particle mass using air fluorescence or a Cherenkov detector array is one of the most difficult task of experimental cosmic ray studies. The information about the primary particle mass is a compound of the produced particle multiplicity, inelasticity, interaction cross-section and many other parameters, thus it is necessary to compare registered showers with sophisticated Monte-Carlo simulation results. In this work we present results of the studies of at least three possible ways of extrapolating protonNucleus and Nucleus-Nucleus cross sections to cosmic ray energies based on the Glauber theory. They are compared with experimental accelerator and cosmic ray data for the proton-air cross section. We also present results of the EAS development with the most popular high-energy interaction models adopted in the CORSIKA program with our cross section extrapolations. The average position of the shower maximum and the width of its distribution are compared with experimental data and some discussion is given.
\end{abstract}

\section{Introduction}

The hadronic interaction cross section is one of the parameters playing a major role during an Extensive Air Shower development. Calculations of hadronic cross sections at ultra high energies require their extrapolation from lower energies where accelerator data are available. Such extrapolations forces the building of a phenomenological models based e.g. on Glauber diffraction theory $[1,2]$. Glauber approximation consists of introducing an eikonal function, $\chi$, representing all phase-shifts related to all possible scattering acts. Eikonal $\chi(\mathbf{b})$ also represents the opacity of two colliding objects. The scattering amplitude in impact parameters space is given by:

$$
\begin{aligned}
& F(s, t)=i \int_{0}^{\infty} J_{0}(b \sqrt{-t}) \Gamma(s, b) b d b= \\
& i \int_{0}^{\infty} J_{0}(b \sqrt{-t})\{1-\exp [-\chi(s, b)]\} b d b,
\end{aligned}
$$

Finally, a knowledge on the form of hadron matter distribution allows for calculations of elastic, inelastic and total cross sections.

$$
\sigma_{t o t}(s)=2 \int\left[1-\mathfrak{R}\left(e^{i \chi(b, s)}\right)\right] d^{2} \mathbf{b}
$$

\footnotetext{
^e-mail: zp@zpk.u.lodz.pl

$\star \star$ e-mail: t.wibig@gmail.com
}

$$
\begin{gathered}
\sigma_{e l}(s)=\int\left[1-e^{i \chi(b, s)}\right]^{2} d^{2} \mathbf{b} \\
\sigma_{\text {inel }}(s)=\int 1-\left[e^{i \chi(b, s)}\right]^{2} d^{2} \mathbf{b}
\end{gathered}
$$

\section{Theoretical description of Proton-Nucleus and Nucleus-Nucleus scattering}

\subsection{Proton - Nucleus scattering}

The essence of the Glauber approximation is the natural assumption that the resulting amplitude phase shift of the collision is the sum of all possible $A$ individual nucleonnucleon phase shifts.

The scattering amplitude is

$$
F(t)=\frac{i}{2 \pi} \int \mathrm{e}^{i \mathbf{i t b}} d^{2} \mathbf{b} \int|\varphi(\{\mathbf{d}\})|^{2}\left\{1-\mathrm{e}^{i \chi_{A}(b,\{\mathbf{d}\})}\right\} \prod_{j=1}^{A} d^{2} \mathbf{d}_{j}
$$

where the function $\varphi$ is the wave-function of the nucleus with the nucleons distribution given by $\{\mathbf{d}\}$. This general formula is a subject of subsequent approximations leading to the set of consecutively simpler equations for the collision cross sections. We assume that there is no space correlation between nucleons. Using a universal nucleon distribution within the nucleus $\rho$ we have 


$$
|\varphi(\{\mathbf{d}\})|^{2}=\prod_{j=1}^{A} \rho_{j}\left(\mathbf{d}_{j}\right)
$$

with the following normalization: $\left(\int \rho_{j}\left(\mathbf{r}_{j}\right) d^{3} \mathbf{r}_{j}=1\right)$.

The next quite obvious approximation is that individual sub-collisions are the same, having universal nucleonnucleon phase-shifts dependences $\chi$. With this we have

$$
\begin{array}{r}
F(t)= \\
\frac{i}{2 \pi} \int \mathrm{e}^{i \mathbf{t b}} d^{2} \mathbf{b}\left\{1-\int \prod_{j=1}^{A} \rho_{j}\left(\mathbf{d}_{j}\right) \mathrm{e}^{i \chi\left(\mathbf{b}-\mathbf{d}_{j}\right)} d^{2} \mathbf{d}_{j}\right\}
\end{array}
$$

On the other hand, the scattering process can be treated as a single collision process with its own nuclear phase shift $\chi_{\text {opt }}(b)$

$$
F(t)=\frac{i}{2 \pi} \int \mathrm{e}^{i \mathbf{t b}}\left\{1-\mathrm{e}^{i \chi_{\mathrm{opt}}(b)}\right\} d^{2} \mathbf{b}
$$

The comparison of Eqs. (7) and (8) leads to the relation between the opacity for the nucleus and the single nucleon:

$$
\mathrm{e}^{i \chi_{\mathrm{opt}}(b)}=\int|\varphi(\{\mathbf{d}\})|^{2} \mathrm{e}^{i \sum_{j=1}^{A} \chi_{j}\left(\mathbf{b}-\mathbf{d}_{j}\right)} d^{2} \mathbf{d}_{j}=\left\langle\mathrm{e}^{i \chi(b,\{\mathbf{d}\})}\right\rangle
$$

To calculate the integral in Eq. 9 we used the fluctuation of the nucleus shape $\rho_{A}(d)$ adopted from Lund model [3] in the form of Woods-Saxon (2-parameter Fermi) distribution leading us to following form of eikonal function:

$$
\chi_{\mathrm{opt}}(b)=i \int d^{2} \mathbf{d} \rho_{A}(d)\left(1-\mathrm{e}^{i \chi(\mathbf{b}-\mathbf{d})}\right)
$$

\subsection{Big nucleus, point nucleon approximation}

We can use the approximation that the number of nucleons in nuclei is relatively big. The number of nucleons (A) can go to infinity keeping the nucleus opacity constant (normalisation). Opacity of the nucleon is the sum of many (small, point-like) scattering centers. Using the optical theorem we obtain:

$$
\chi_{\mathrm{opt}}(b)=\frac{1}{2} \sigma_{\mathrm{pp}}^{\mathrm{tot}} \rho_{A}(b)
$$

This can be substituted in Eq. 4 and the inelastic cross section can be described by:

$$
\sigma_{\text {inel }}^{p A}=\int\left\{1-\left[1-\sigma_{\mathrm{pp}}^{\mathrm{tot}} \frac{\rho_{A}(b)}{A}\right]^{A}\right\} d^{2} \mathbf{b} .
$$

\subsection{Nucleus - Nucleus scattering}

The treatment of nucleon-nucleus presented above can be extended to the case of nuclei collisions with the amplitude defined as

$$
F(t)=\frac{i}{2 \pi} \int \mathrm{e}^{i \mathbf{t b}} d^{2} \mathbf{b}\left\{1-\int d^{2} \mathbf{d}_{i} \rho_{\mathrm{AB}}\left(\mathbf{d}_{i}\right) \mathrm{e}^{i \chi\left(\mathbf{b}-\mathbf{d}_{i}\right)}\right\}
$$

Using Eq.(8) we can define the overall nucleus-nucleus opacity

$$
\mathrm{e}^{i \chi \mathrm{AB}(b)}=\int d^{2} \mathbf{d}_{i} \rho_{\mathrm{AB}}(\{\mathbf{d}\}) \mathrm{e}^{i \sum_{i=1}^{A} \chi\left(\mathbf{b}-\mathbf{d}_{i}\right)}=\left\langle\mathrm{e}^{i \chi\left(b,\left\{\mathbf{d}_{\mathbf{A}}\right\},\left\{\mathbf{d}_{\mathbf{B}}\right\}\right)}\right\rangle
$$

Thus the nucleus-nucleus scattering amplitude is, with the analogy to Eq.(8)

$$
F(\mathbf{t})=\frac{i}{2 \pi} \int \mathrm{e}^{i \mathbf{t b}}\left(1-\mathrm{e}^{i \chi_{A B}(b)}\right) d^{2} \mathbf{b} .
$$

The "big nucleus" and "point nucleon" approximation can also be used in this case leading to

$$
\sigma_{\text {inel }}^{A B}=\int\left\{1-\left[1-\sigma_{\mathrm{pp}}^{\mathrm{tot}} \frac{\rho_{A B}(b)}{A B}\right]^{A B}\right\} d^{2} \mathbf{b} .
$$

\subsection{Probabilistic framework}

One of the existing ways in the literature to describe nucleus-nucleus interactions is the probabilistic formalism (see, e.g., [4]). It assumes that the collision between individual nucleons of colliding nuclei are not correlated and do not interfere with each other.

If there are $A B$ pairs of nucleons which could take part in the interaction the probability of having $n$ inelastic interactions is

$$
p(n, b)=\left(\begin{array}{c}
A B \\
n
\end{array}\right)\left[\frac{\rho_{A B}(b)}{A B} \sigma_{\text {inel }}^{p p}\right]^{n}\left[1-\frac{\rho_{A B}(b)}{A B} \sigma_{\text {inel }}^{p p}\right]^{A B-n},
$$

The summation over $n$ can be performed and the result with the integration over $b$ gives the value of the so-called "production cross section":

$$
\sigma_{\text {inel }}^{A B}=\int d^{2} \mathbf{b}\left\{1-\left[1-\frac{\rho_{A B}(b)}{A B} \sigma_{\text {inel }}^{p p}\right]^{A B}\right\},
$$

which is quite similar to the result in Eq.(16), but with $\sigma_{\text {inel }}$ in place of $\sigma_{\text {tot }}$.

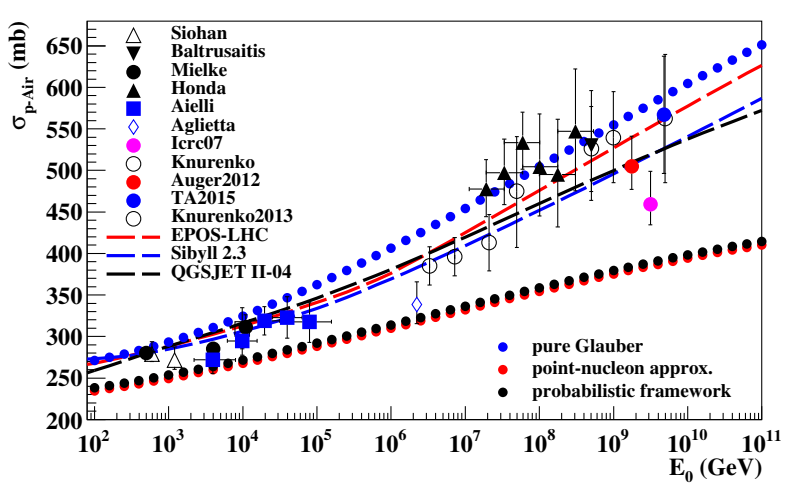

Figure 1. p-Air inelastic cross-section calculated using Glauber theory, point-nucleon approximation and probabilistic framework compared with experimental data [5] 


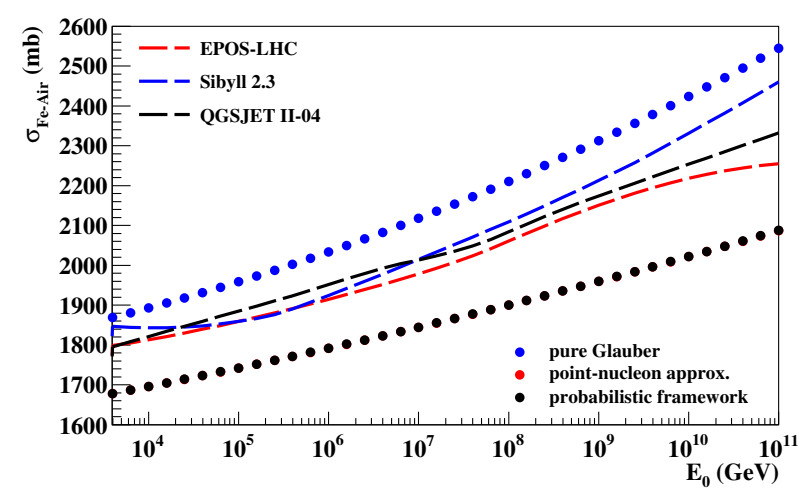

Figure 2. Fe-Air inelastic cross-section calculated using Glauber theory, point-nucleon approximation and probabilistic framework.

\section{Calculated $\sigma_{\text {inel }}$ Cross-Sections}

We present inelastic cross sections calculated using three previously discussed ways. Cross sections for p-Air and Fe-Air collisions are presented separately in Figs 1 and 2 respectively. In the case of p-Air cross-section, results are presented with available experimental data. Results in both cases are compared with cross-section values currently existing in three high-energy interaction models CORISKA - EPOS-LHC [6], Sibyll 2.3c [7, 8] and QGSJETII-04 [9].

The basis of the approach to the calculation of inelastic Proton-Nucleus and Nucleus-Nucleus cross sections is more precisely described in section 2 [10].

\section{Simulations of $X_{\max }$ parameter}

We used the calculated $\sigma_{\text {inel }}$ cross section in the CORSIKA program for simulations of the longitudinal development of an EAS. Simulations were performed using three quite popular high-energy intraction models - EPOS-LHC, QGSJETII-04 and Sibyll 2.3c. As a result we present plots of $X_{\text {max }}$ position versus primary energy for each case compared with results for cross sections currently existing in CORSIKA and experimental data (Fig. 3). Additionally we present RMS parameters of calculated values. The following plots concerns EAS simulations with modified $\sigma_{p N}^{i n e l}$ and $\sigma_{N N}^{i n e l}$ cross sections, not modifications of high energy models. All simulations have been performed using the NKG lateral disribution function. Simulations in 4 points on energy scale consist of the following statistics: $10^{11} \mathrm{GeV}$ - 100 showers, $10^{10} \mathrm{GeV}-250$ showers, $10^{9}$ $\mathrm{GeV}$ - 500 showers, $10^{8} \mathrm{GeV}$ - 500 showers. Results are compared with experimental data [11-14] (Fig. 3).

The presented results of $X_{\max }$ parameters concerns simulations with new inelastic cross sections instead of those originally existing in the EPOS-LHC, QGSJETII-04 and Sibyll 2.3c models. Implementation of our cross sections was only one modification made in CORSIKA for these simulations.
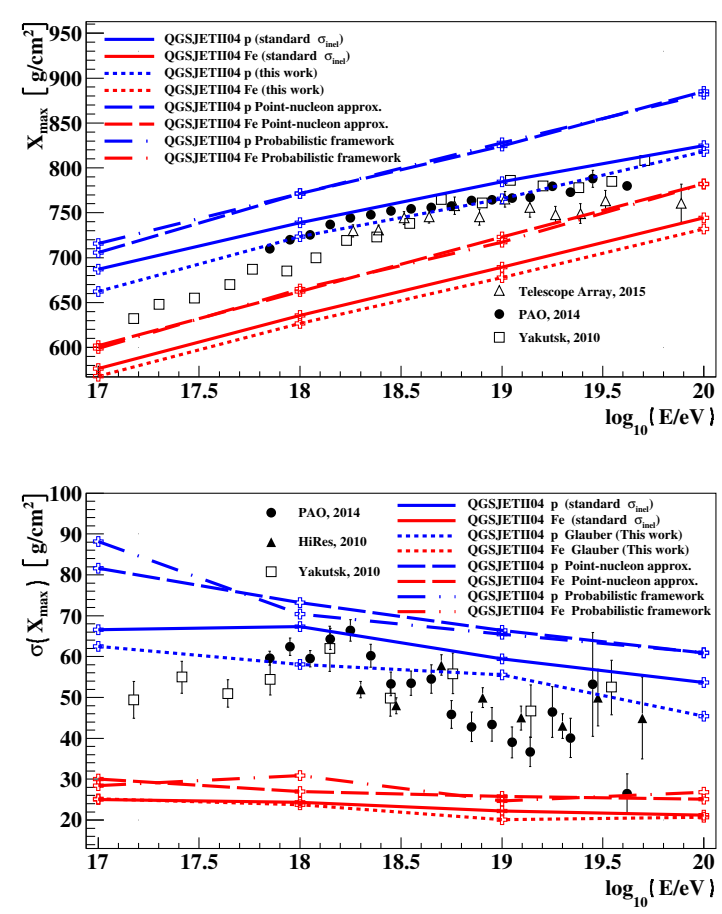

Figure 3. (top): Position of $X_{\max }$ simulated using QGSJETII-04 model with different $\sigma_{\text {inel }}$. Results for our Glauber calculations (dotted line) are compared with point-nucleon and probabilistic approximations (dashed lines) and original $\sigma_{\text {inel }}$ in QGSJETII04 model (solid line), (bottom): $\sigma$ parameter of obtained $X_{\max }$ values.

\section{Conclusions}

We present revised results of inelastic cross-sections $\left(\sigma_{\text {inel }}\right)$ calclations based on the Glauber diffraction theory and point-nucleon and probabilistic approximations. Differences between results of Glauber theory and approximations are increasing with energy. For all discussed models our $\sigma_{\text {inel }}$ in the Glauber case are a little bit higher than commonly used in CORSIKA (Fig. 1). It forces faster EAS development than we can observe in results of $X_{\max }$ simulations (Figs. 3 - 5). Simulations with new Glauber $\sigma_{\text {inel }}$ provides a better agreement with measured $X_{\text {max }}$ for protons as the primary particle. Simulated $X_{\max }$ values have been obtained using CORSIKA with NKG option only. In future the presented $\sigma_{\text {inel }}$ cross sections will be implemented in CONEX [15] code and simulations will be repeated with EGS and higher statistics. Exact determination of nuclear cross-section is very difficult because of a lack of knowledge about nucleus hadron matter distribution especially at the highest energies. This kind of consideration is very important for correct interpretation of cosmic ray data.

Acknowledgments: This work was supported by grant no 2016/20/T/ST9/00589 funded by the National Science Centre in Poland. 

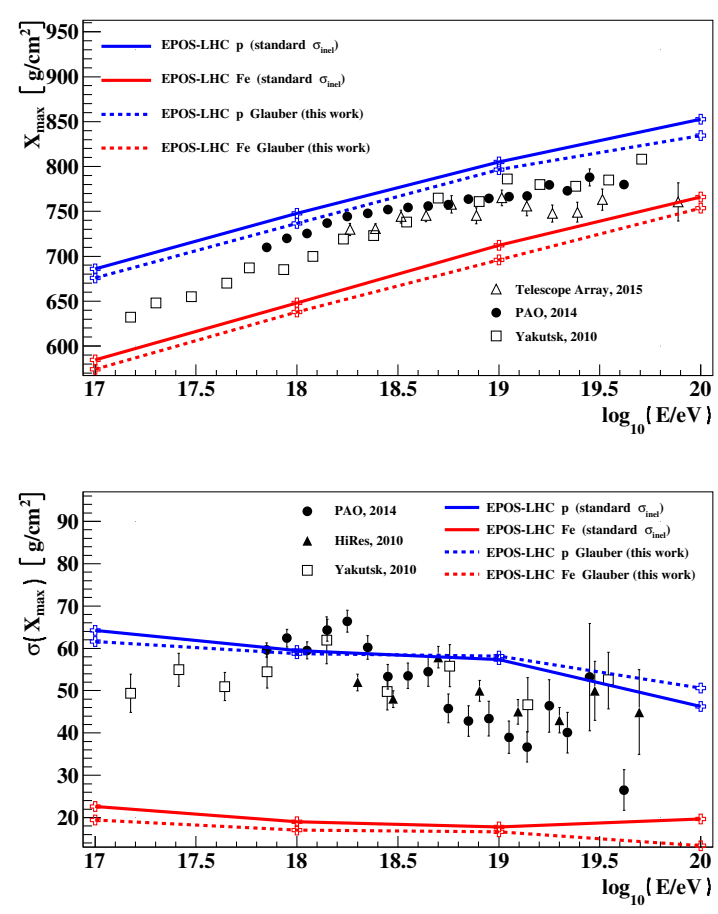

Figure 4. (top): Position of $X_{\max }$ simulated using EPOS-LHC model with different $\sigma_{\text {inel }}$. Results for our Glauber calculations (dotted line) are compared original $\sigma_{\text {inel }}$ in EPOS-LHC model (solid line), (bottom): $\sigma$ parameter of obtained $X_{\max }$ values.
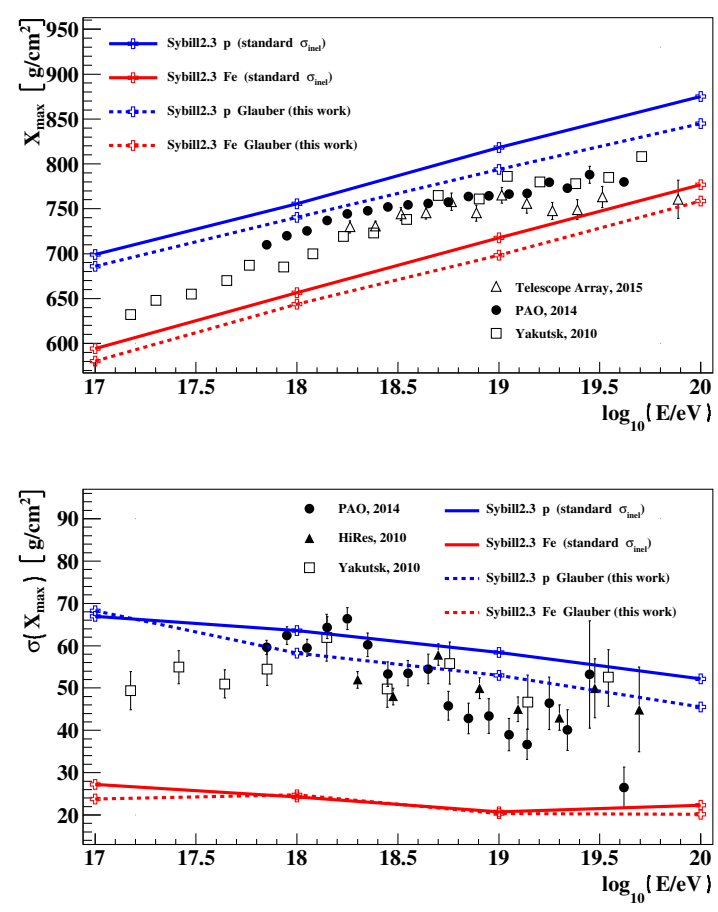

Figure 5. (top): Position of $X_{\max }$ simulated using Sibyll 2.3c model with different $\sigma_{\text {inel }}$. Results for our Glauber calculations (dotted line) are compared with original $\sigma_{\text {inel }}$ in Sibyll $2.3 \mathrm{c}$ model (solid line), (bottom): $\sigma$ parameter of obtained $X_{\max }$ values.

\section{References}

[1] R. Glauber et al., Interscience, New York pp. 1 - 315 (1959), ed. WE Britin and LG Dunham

[2] R. Glauber, G. Matthiae, Nuclear Physics B 21, 135 (1970)

[3] L. Ding, E. Stenlund, Computer Physics Communications 59, 313 (1990)

[4] J. Engel, T.K. Gaisser, P. Lipari, T. Stanev, Phys. Rev. D 46, 5013 (1992)

[5] R. Ulrich, J. Blümer, R. Engel, F. Schüssler, M. Unger, New Journal of Physics 11, 065018 (2009)

[6] T. Pierog, I. Karpenko, J.M. Katzy, E. Yatsenko, K. Werner, Phys. Rev. C 92, 034906 (2015)

[7] F. Riehn, H.P. Dembinski, R. Engel, A. Fedynitch, T.K. Gaisser, T. Stanev, PoS ICRC2017, 301 (2018), 1709.07227

[8] E.J. Ahn, R. Engel, T.K. Gaisser, P. Lipari, T. Stanev, Phys. Rev. D 80, 094003 (2009)

[9] S. Ostapchenko, Phys. Rev. D 83, 014018 (2011)

[10] Z. Plebaniak, T. Wibig, PoS ICRC2017, 581 (2018)

[11] A. Aab, P. Abreu, M. Aglietta, E.J. Ahn, I. Al Samarai, I.F.M. Albuquerque, I. Allekotte, J. Allen, P. Allison, A. Almela et al. (Pierre Auger Collaboration), Phys. Rev. D 90, 122005 (2014)

[12] R. Abbasi, M. Abe, T. Abu-Zayyad, M. Allen, R. Anderson, R. Azuma, E. Barcikowski, J. Belz, D. Bergman, S. Blake et al., Astroparticle Physics 64, 49 (2015)

[13] R.U. Abbasi, T. Abu-Zayyad, M. Al-Seady, M. Allen, J.F. Amman, R.J. Anderson, G. Archbold, K. Belov, J.W. Belz, D.R. Bergman et al. (The High Resolution Fly's Eye Collaboration), Phys. Rev. Lett. 104, 161101 (2010)

[14] S.P. Knurenko, A. Sabourov, Astrophysics and Space Sciences Transactions 7, 251 (2011)

[15] T. Pierog, M. Alekseeva, T. Bergmann, V. Chernatkin, R. Engel, D. Heck, N. Kalmykov, J. Moyon, S. Ostapchenko, T. Thouw et al., Nuclear Physics B - Proceedings Supplements 151, 159 (2006), Very High energy Cosmic Ray Interactions 